\title{
Modeling of target strength of swimbladder fish at high frequencies
}

\author{
Zhen $\mathrm{Ye}^{*}$ and Masahiko Furusawa** \\ *Institute of Ocean Sciences, \\ P.O. Box 6000, 9860 W. Saanich Rd., Sidney, British Columbia, Canada \\ **National Research Institute of Fisheries Engineering, \\ Ebidai, Hasaki, Kashima, Ibaraki, 314-04 Japan
}

(Received 7 March 1995)

\begin{abstract}
An aim of this paper is to compare a proposed simple model for the target strength (TS) of swimbladder fish with existing models. Through the comparison we verify the validity of the simple model. Particularly, two more sophisticated models are compared with: namely, the exactly solvable soft spheroid model and the ray-Kirchhoff approximate model. The comparisons show that at high frequencies or intermediate frequencies the present model can yield results in reasonable agreement with that from the other two models respectively. Indirect comparison with experimental data is also briefly made. It is shown that the model may facilitate general calculation of the target strength of swimbladder fish.
\end{abstract}

Keywords: Sound scattering, Fisheries acoustics, Target strength, Modeling

PACS number: $43.30 . \mathrm{Gv}$

\section{INTRODUCTION}

Fish target strength (TS) has long been one of most important components in fisheries acoustics research. Theoretical models seem to be imperative as they can guide interpolation and/or extrapolation of limited experimental data so as to deduce general trend for fish target strength. ${ }^{1)}$ Moreover, model study can help design echo sounder for optimal results, ${ }^{2)}$ and improve quantifying fish stocks in situ and cast lights on species recognition.

To date, many theoretical models for fish target strength have been used. Roughly speaking, models can be classified into two categories: models for low frequency scattering and models for relatively high frequency scattering. For the low frequencies, a spherical gas bubble has often been used to represent the fish swimbladder. Different emphases on the fish flesh have led to various models for swimbladder fish such as air bubble in an infinite elastic medium, ${ }^{3)}$ air bubble in a rubberlike shell, ${ }^{\text {) }}$ air bubble in a fluid shell ${ }^{5)}$ and air bubble in an elastic shell. ${ }^{6}$ ) For the high frequency cases, the fish swimbladder was shown to scatter the most acoustic energy. ${ }^{1,7)}$ Therefore, the main attention has been focused on calculating TS of the swimbladder. In this paper we will mainly focus on the high frequency acoustic scattering. Deduced from the Helmholtz-Kirchhoff integral (which is formally one of the exact solutions to sound scattering problem), the Kirchhoff approximation was used by Foote ${ }^{8)}$ to calculate the TS of fish swimbladders having irregular geometry. As demonstrated by Foote in his comparison to experimental data, the Kirchhoff approximation appears to be a powerful method for the purpose at rather high frequencies. However, in this method to exactly obtain the surface area elements of a swimbladder is often laborious if not impossible. To deduce the general TS trend of swimbladder fish, Furusawa ${ }^{1)}$ proposed to model swimbladder as a 
prolate spheroid. A merit of his method is that the solution is exact and can thus provide a check for other approximation schemes developed for exotic models. Based on the exact modal series solution to an infinitely long cylinder, Junger ${ }^{9)}$ and Stanton ${ }^{10)}$ proposed a deformed cylinder model to study the sound scattering from finite elongated objects. The deformed cylinder model has been claimed to be useful to study the TS of liquid marine organisms such as krill. This method has merit in that it may be used for modeling irregularly elongated fish swimbladder with less parameters required, as we shall show in this paper. In this method, the shape of the fish swimbladder may be relatively easy to obtain by using soft X-ray analysis.

In this paper, we will examine the deformed cylinder model for acoustic scattering from an elongated fluid scatterer, since many fish swimbladders resemble elongated form. The model will be modified for easy calculation of the TS of a fish swimbladder. A simple formula will be derived for the acoustic scattering function. Detailed comparisons with the existing models such as the spheroid model ${ }^{1)}$ and a ray-Kirchhoff model ${ }^{11)}$ will be presented. Limitations to the present simple model will also be addressed.

\section{FORMULATION OF THE PROBLEM}

We first briefly review the sound scattering from an infinitely long fluid cylinder for which the exact solution exists in the literature. Then we examine the approximations taken by Junger ${ }^{9)}$ and Stanton ${ }^{10)}$ to study the sound scattering from an elongated fluid cylinder of finite length. Simplification to their approximation will be presented at the end of this section with limitations to their approximation being addressed.

The sound scattering from an infinitely long cylinder can be formulated into solving the following wave equation

$$
\left(\nabla^{2}+k^{2}\right) p=0, \quad k=\omega / c_{\mathrm{w}}
$$

with appropriate boundary conditions,

$$
p_{\text {ine }}+p_{\text {seat }}=p_{\text {int }},
$$

(continuation of pressure at the boundary) and

$$
u_{r_{\text {ine }}}+u_{r_{\text {seat }}}=u_{r_{\mathrm{int}}},
$$

(continuation of radial velocities at the boundary) where inc, scat and int are respectively representing the incident, scattered and internal quantities. In the above, $\omega$ is the angular frequency and $c_{\mathrm{w}}$ is the sound speed in water. Assuming the incident wave as $p_{\text {inc }}=P_{0} \exp (i k r)$, simple calculation leads to the following solution for the scattered pressure wave in the cylindrical coordinates,

$$
p_{\text {seat }}=P_{0} \sum_{m=0}^{\infty} B_{m}(a) \cos (m \phi) H_{m}^{(1)}(k r),
$$

where $H_{m}{ }^{(1)}(x)$ is the Hankel function of the first kind, $\phi$ is the azimuthal angle that sweeps through the plane perpendicular to the longitudinal cylinder axis and $r$ is the distance from the axis. The above coefficients $B_{m}(a)$ are determined by

$$
B_{m}(a)=-\epsilon_{m} i^{m} /\left[1+i C_{m}\left(a, k, k^{\prime}\right)\right]
$$

with $\epsilon_{m}$ being the Neumann factor and,

$$
C_{m} \equiv \frac{\left[J_{m}{ }^{\prime}\left(k^{\prime} a\right) N_{m}(k a)\right] /\left[J_{m}\left(k^{\prime} a\right) J_{m}{ }^{\prime}(k a)\right]-g h\left[N_{m}{ }^{\prime}(k a) / J_{m}{ }^{\prime}(k a)\right]}{\left[J_{m}{ }^{\prime}\left(k^{\prime} a\right) J_{m}(k a)\right] /\left[J_{m}\left(k^{\prime} a\right) J_{m}{ }^{\prime}(k a)\right]-g h},
$$

$$
g=\rho_{\mathrm{g}} / \rho_{\mathrm{w}}, \quad h=c_{\mathrm{g}} / c_{\mathrm{w}}, \quad k^{\prime}=\omega / c_{\mathrm{g}},
$$

where $J_{m}(x)$ and $N_{m}(x)$ are the first kind Bessel function and Neumann function respectively, $a$ is the radius of the cylinder, $\rho_{\mathrm{g}}$ and $c_{\mathrm{g}}$ are density and sound speed inside the scatterer, and $J_{m}{ }^{\prime}(x)$ and $N_{m}{ }^{\prime}(x)$ represent $\partial J_{m}(x) / \partial x$ and $\partial N_{m}(x) / \partial x$ respectively. We note that we have assumed the normal incidence.

To make the above solution usable for a finite cylinder, Junger ${ }^{9)}$ and Stanton ${ }^{10)}$ proposed that the solution in Eq. (4) be written in an integral form and the integration be performed along the longitudinal axis of the cylinder, e.g.,

$$
p_{\mathrm{scat}}=\int_{-\infty}^{\infty} d z Q(z, a, r),
$$

where $z$ is the coordinate along the longitudinal axis. The integrand $Q(z, a, r)$ can be determined by equating Eq. (6) to Eq. (4):

$$
\begin{aligned}
& \int_{-\infty}^{\infty} d z Q(z, a, r) \\
& \quad=P_{0} \sum_{m=0}^{\infty} B_{m}(a) \cos (m \phi) H_{m}{ }^{(1)}(k r) .
\end{aligned}
$$

The difference between Junger's and Stanton's approach can be said as follows (Refer to Fig. 1(b) 


\section{Z. YE and M. FURUSAWA: MODELING OF FISH TARGET STRENGTH}

and (c)): In Junger's approach, the differential elements are cylinders; but in the Stanton's approach, the differential elements are line elements. Stanton approximated the elongated object as a line scatterer.

In the Stanton's approach, function $Q(z, a, r)$ is found to be in the following form:

$$
Q(z, a, r) \sim \frac{e^{i k r_{\mathrm{s}}}}{r_{\mathrm{s}}},
$$

with

$$
r_{\mathrm{s}}=\sqrt{r^{2}+z^{2}} .
$$

The scattering pressure wave for a finite cylinder is heuristically obtained by cutting the integration interval from $(-\infty, \infty)$ to $(-L / 2, L / 2)$ for a cylinder of length $L$. Then the scattering wave from a finite cylinder is written as

$$
p_{\text {scat }}=\int_{-L / 2}^{L / 2} d z Q(z, a, r) .
$$

Strictly speaking, in this equation the radius $a$ must be constant (straight cylinder). However, in order to calculate scattering function from a slender body of revolution Junger ${ }^{9)}$ relaxed this requirement. Therefore the scattering function can be written as

$$
p_{\text {scat }}=\int_{-L / 2}^{L / 2} d z Q(z, a(z), r) .
$$

The value of function $Q(z, a(z), r)$ at $z$ is determined by

$$
\begin{aligned}
& \int_{-\infty}^{\infty} d z_{1} Q\left(z_{1}, a(z), r\right) \\
& \quad=P_{0} \sum_{m=0}^{\infty} B_{m}(a(z)) \cos (m \phi) H_{m}{ }^{(1)}(k r) .
\end{aligned}
$$

Junger $^{9)}$ applied this approximation to a prolate spheroid rigid scatterer, yielding encouraging results.

As we see, up to this stage the results are limited to the normal incidence. To overcome this problem, Stanton $^{10)}$ first wrote $Q(z, a, r)$ in a form like,

$$
Q(z, a, r) \sim \frac{e^{i k_{\mathrm{s}} r_{\mathrm{s}}}}{r_{\mathrm{s}}},
$$

and then added an adjustable phase factor $\epsilon_{\mathrm{s}}$ so that when applied to a finite rigid straight-cylinder the approximation can recover to the well-known result

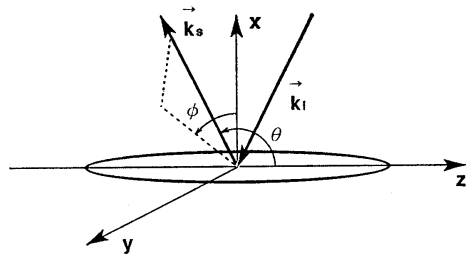

(a) (b)

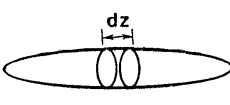

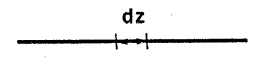

(c)
Fig. 1 Geometry for modeling: a) Geometry for an elongated scatterer; b) The difierential elements in Junger's approach; c) The differential elements in Stanton's approach.

for a finite rigid straight-cylinder. ${ }^{12)}$ It is found that $\epsilon_{\mathrm{s}}$ can be determined implicitly by

$$
\begin{array}{rl}
\int_{-L / 2}^{L / 2} & d z \frac{e^{i k\left(r_{\mathrm{s}}+\varepsilon_{\mathrm{s}}\right)}}{r_{\mathrm{s}}} \\
& \sim \frac{e^{i k r}}{r} \int_{-L / 2}^{L / 2} d z e^{i\left(\vec{k}_{\mathrm{i}}-\vec{k}_{\mathrm{s}}\right) \cdot \vec{z}},
\end{array}
$$

where $\vec{k}_{\mathrm{i}}, \vec{k}_{\mathrm{s}}\left(k_{\mathrm{i}}=k_{\mathrm{s}}=k\right)$ are wave vectors for incident and scattered waves respectively and $r$ is the distance from the center of the scatterer to the observing point (not to be confused with the $r$ used earlier). The resulting $Q(z, a(z), r)$ is then taken into Eq. (9) to obtain the scattering wave. By writing this scattering wave at far field $k r \gg 1$ as

$$
p_{\mathrm{scat}}=f\left(\vec{k}_{\mathrm{i}}, \vec{k}_{\mathrm{s}}\right) \frac{e^{i k r}}{r},
$$

the scattering function $f\left(\vec{k}_{\mathrm{i}}, \vec{k}_{\mathrm{s}}\right)$ can be determined as,

$$
\begin{aligned}
f\left(\vec{k}_{\mathrm{i}}, \theta, \phi\right)= & \frac{-i}{\pi} \int_{-L / 2}^{L / 2} d z \sum_{m=0}^{\infty} B_{m}(a(z)) \\
& \cdot(-i)^{m} \cos (m \phi) e^{i\left(\overrightarrow{k_{\mathrm{i}}}-\overrightarrow{k_{\mathrm{s}}}\right) \cdot \vec{z}}
\end{aligned}
$$

where $\theta, \phi$ are scattering angles (Fig. 1(a)). We note that for the case when the incidence is not normal, the coefficients $C_{m}$ in Eq. (5) should be changed into

$$
C_{m} \equiv \frac{\left[J_{m}{ }^{\prime}\left(K^{\prime} a\right) N_{m}(K a)\right] /\left[J_{m}\left(K^{\prime} a\right) J_{m}{ }^{\prime}(K a)\right]-g h\left[N_{m}{ }^{\prime}(K a) / J_{m}{ }^{\prime}(K a)\right]}{\left[J_{m}{ }^{\prime}\left(K^{\prime} a\right) J_{m}(K a)\right] /\left[J_{m}\left(K^{\prime} a\right) J_{m}{ }^{\prime}(K a)\right]-g h}
$$


in which $K=k \cos \theta$ and $K^{\prime}=k^{\prime} \cos \theta$, where $\theta$ is the tilt angle of incidence.

For backscattering, $\phi=\pi$, and $\vec{k}_{\mathrm{s}}=-\vec{k}_{\mathrm{i}}$. So the backscattering function now can be written as,

$$
\begin{aligned}
f_{\mathrm{bs}}=f\left(\vec{k}_{\mathrm{i}}, \theta, \pi\right)= & \frac{-i}{\pi} \int_{-L / 2}^{L / 2} d z \sum_{m=0}^{\infty}(-1)^{m} B_{m}(a(z)) \\
& \cdot(-i)^{m} e^{2 i \vec{k}_{\mathrm{i}} \cdot \vec{z}} .
\end{aligned}
$$

This equation has been used successfully to calculate the TS of liquid-filled marine organisms such as krill etc.

From the above derivation, we see that the Stanton's extension from normal incidence to the incidence with a tilt angle is rather ad hoc. As shown later, the Stanton's extension may be only valid for the incidence of small tilt angle. In addition, from Eq. (13) we see that the tilt angle of the incidence will only enter the factor $C_{m}$. This may imply that the present extension may not be appropriate for bistatic scattering. In a separate paper, we will propose a more strict approach for the incident at an tilt angle. Nevertheless, in the following we will use Stanton's approach for calculating the TS of a fish swimbladder and derive our own model.

Consider sound scattering from fish swimbladder. Since most echo sounders for fish TS measurements are set at rather high frequencies (short wavelength compared to the size of fish swimbladder), one may approximate $B_{m}(a(z))$ as ${ }^{13)}$ :

$$
\begin{aligned}
& \sum_{m=0}^{\infty} B_{m}(a(z))(-i)^{m} \\
& \quad \approx\left(\frac{\pi}{4} k a(z) \sin \left(\theta_{z}\right)\right)^{1 / 2} e^{-2 i k a(z) \sin \left(\theta_{z}\right)},
\end{aligned}
$$

where $\theta_{z}$ is the angle between $\vec{z}$ and $\vec{k}_{\mathrm{i}}$. Moreover, for a deformed swimbladder whose longitudinal axis is not straight, we assume that the following modification holds,

$$
\int_{-L / 2}^{L / 2} d z[\cdot] \approx \int_{-L / 2}^{L / 2} d \vec{z}[\cdot]
$$

With Eqs. (15) and (16), we derive that the final scattering function for a fish swimbladder can be written as

$$
\begin{aligned}
f_{\mathrm{bs}}= & \frac{-i}{2 \pi^{1 / 2}} \int_{-L / 2}^{L / 2} d \vec{z}\left[k a(z) \sin \left(\theta_{z}\right)\right]^{1 / 2} \\
& \cdot \exp \left[-2 i k a(z) \sin \left(\theta_{z}\right)+2 i \vec{k}_{\mathrm{i}} \cdot \vec{z}\right],
\end{aligned}
$$

which is our simplified backscattering function for an elongated swimbladder stemmed from Stanton's approach. Since Eq. (17) is rather simple, the formula can be easily programmed in, for example, Metlab etc.

In practical usage of Eq. (17), one may just need the following measurements on fish swimbladder: 1) from ventral and lateral $X$-rays of fish we can obtain the revolution of the radius of the cross section along the longitudinal axis; 2) On the Xray side view of fish swimbladder, one can draw roughly a straight line from the head end to the tail end. This line is used as a reference axis; 3) Dissecting the swimbladder into a series of small cylindrical elements, we can measure the approximate radius and length of each element. We can also measure the deviation of the center of each element from the reference axis to determine the bending of swimbladder. Taking these into Eq. (17), we can easily calculate backscattering function for an elongated swimbladder.

As a check of Eq. (17), we consider a finite uniform cylinder. The scattering function can be calculated from Eq. (17) as,

$$
f_{\mathrm{bs}}(\theta)=\frac{-i}{2 \pi^{1 / 2}}(k a \cos \theta)^{1 / 2} e^{-2 i k a \cos \theta} L \frac{\sin (k L \sin \theta)}{k L \sin \theta},
$$

where $\theta$ is the incidence angle. The backscattering cross section is given by

$$
\sigma_{\mathrm{bs}}(\theta)=\frac{1}{4 \pi}(k a \cos \theta) L^{2}\left(\frac{\sin (k L \sin \theta)}{k L \sin \theta}\right)^{2},
$$

which can be found in many textbooks such as Ref. 12).

\section{NUMERICAL RESULTS}

In this section, we will apply Eq. (17) to existing swimbladder models and compare the results to the results from other approaches.

The target strength of a scatterer is defined as follows,

$$
T S \equiv 10 \log \left[\left|f_{\mathrm{bs}}\right|^{2}\right] .
$$

In practice, the reduced target strength is often used for convenience. It is defined as,

$$
T S_{\mathrm{r}} \equiv 10 \log \left[\left|f_{\mathrm{bs}} / L\right|^{2}\right],
$$

where $L$ denotes the length of scatterer. In the present case, $L$ is the fork length of a fish.

If it is the buoyancy that mainly balances the weight of a swimbladder fish, then the swimbladder length will be roughly 0.34 of the fish fork-length for many kinds of fish such as Japanese mackerel (Scomber japonicus) and sea bream (Chrysophrys 
major). ${ }^{1)}$ By assuming this geometry for fish swimbladders, the general trend of many target strengths of fish can be predicted. However, in this paper we will limit ourselves to illustrative comparisons with existing model results.

Two comparisons will be made. One is with Furusawa's spheroidal model ${ }^{1)}$ and the other is with the model by Clay and Horne. ${ }^{11)}$ The reasons for such comparisons are as follows. Firstly, Furusawa's spheroidal model has exact solution. The comparison with the exact solution will paint us a clear picture about how good or how bad the simple model would be. Secondly, to our best knowledge, Clay and Horne's paper is the first paper that has clear X-ray pictures of fish (Atlantic cod) with fish shapes and swimbladder structures being outlined relatively precisely. Applying the present model to their fish will provide first hand information of the practical use of the model as compared with other independent approximate methods. In their forthcoming paper, McClatchie et al. ${ }^{14)}$ will make eye to eye comparisons of the model in Eq. (17) with other two models, namely the Kirchhoff model $^{8)}$ and the equi-cylinder model, ${ }^{15)}$ on three New Zealand commercial fish species. It seems that the model in Eq. (17) can yield reasonable results as compared with the other two. However, in the study the shapes of fish swimbladders are obtained by plaster casts, which may have changed the natural shape of fish swimbladders making the results biased.

\subsection{Comparison with Furusawa's Spheroidal Model}

A spheroidal scatterer can be depicted as in Fig. 2. In Furusawa's notation, ${ }^{1)}$ the reduced target strength is defined as,

$$
T S_{\mathrm{r}} \equiv 20 \log [|f /(2 a)|],
$$

where $a$ is the major radius of the prolate spheroid. In his study, Furusawa has modeled fish swimbladder as a spheroid, which appears to be a reasonable assumption for high frequencies. ${ }^{1)}$ The scattering function can be calculated by Eq. (2) of Ref. 1). To compare with Furusawa's results, $a$ is taken as 3.4 $\mathrm{cm}$ (i.e. $L=20 \mathrm{~cm}$ ). First we consider the normal incidence. We take the aspect ratio to be 0.15 . The reduced TS is plotted against $k a$ in Fig. 3(a), in which dashed line represents the TS at optical limit $\left(T S=10 \log a^{2} / 4\right)^{12)}$ and the dotted-dashed line is

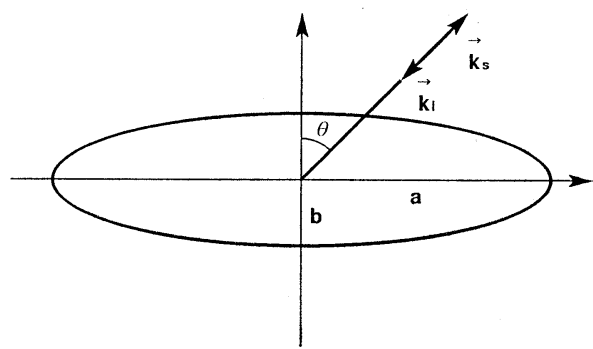

Fig. 2 Geometry for a spheroidal scatter. Here $a$ is the major axis, and $b$ is the minor axis. The incidence angle is indicated in the figure. The direction of the backscattered wave is opposite to this incidence. The aspect ratio is defined as $b / a$.
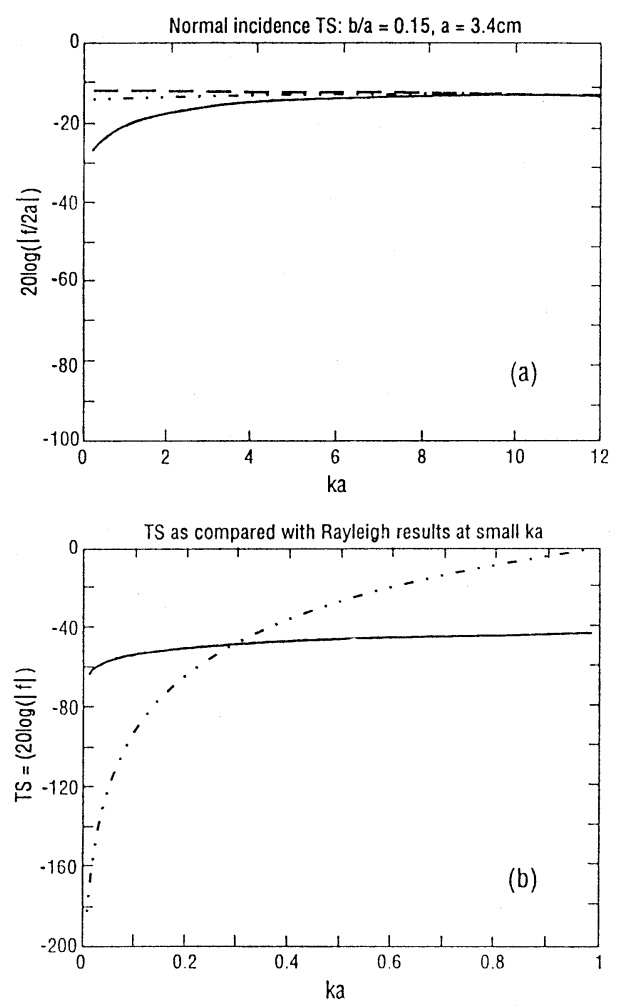

Fig. 3 Reduced TS vs. ka: a; Comparison with Furusawa's result for a spheroid with the axis aspect ratio being 0.15 at normal incidence. In the diagrams, the dashed line is theoretical limit and the dotted-dashed line is from Fig. 3(a) in Furusawa $^{1)}$; b) Comparison with Rayleigh scattering law for small $k a$. 
from Furusawa. ${ }^{1)}$ As shown in Fig. 3(a), the present result seems agreeable to the Furusawa's result for $k a>6$. However, the discrepancy remains for low frequency region. As $k a$ decreases, our results do have the tendency to drop. However, they do not follow Rayleigh scattering law for low ka. This is shown in Fig. 3(b). Hence the present model cannot be used for low $k a$ cases where the Rayleigh law applies $(k a \ll 1)$. Rayleigh scattering says that the scattering cross section is proportional to $k^{4}$.

We also inspect TS $v s$. tilt angles. In Fig. 4, we plot several reduced TS vs. tilt angles at different aspect ratios and various $k a$, as compared to Furusawa's results. From Fig. 4, the following general trends can be said: 1) At given aspect ratio, the oscillatory features will appear with increasing $k a$ in the present model, which seem to be absent in Furusawa's result; 2) For small $k a$, the tilt angle dependence of the reduced TS seem to agree with that of Furusawa's for up to 70 degrees of tilt angle. However, the present model constantly predicts lower TS than spheroidal model (Fig. 4(a)); 3) With increasing $k a$, the present results agree to that of Furusawa only for small tilt angle scattering (e.g. $<20$ degrees, Refer to Fig. 4(b) and (c)). 4) The present theory cannot be used for end-on incidence, in which the TS drops more rapidly than expected. At the end-on incident, the target strength calculated from the radii of curvature is $\left.10 \log \left[\left(b^{2} / a\right)^{2} / 4\right],{ }^{12}\right)$ which gives the reduced TS as $-45 \mathrm{~dB}$.

Over all, the present model can predict similar results as the Furusawa's model for large $k a$ and at the near normal incidence.

\subsection{Comparison with the Results of Clay and Horne}

The definition of reduced TS has been given in Eq. (19). To compare with Clay and Horne's results, ${ }^{11)}$ we choose cod D in their Fig. 2 as an example. The X-ray picture of the fish is given in their Fig. 1 (reproduced in Fig. 5(a)), in which both ventral and lateral X-rays of live Atlantic cod was given. As we can see from their Figs. 1 and 2, the cross sections of the fish swimbladder can be roughly modeled as circular shapes having different averaged radii along the longitudinal axis.

By digitizing the fish swimbladder into a series of consecutive small cylinders, the total scattering function can be evaluated from Eq. (17). As shown
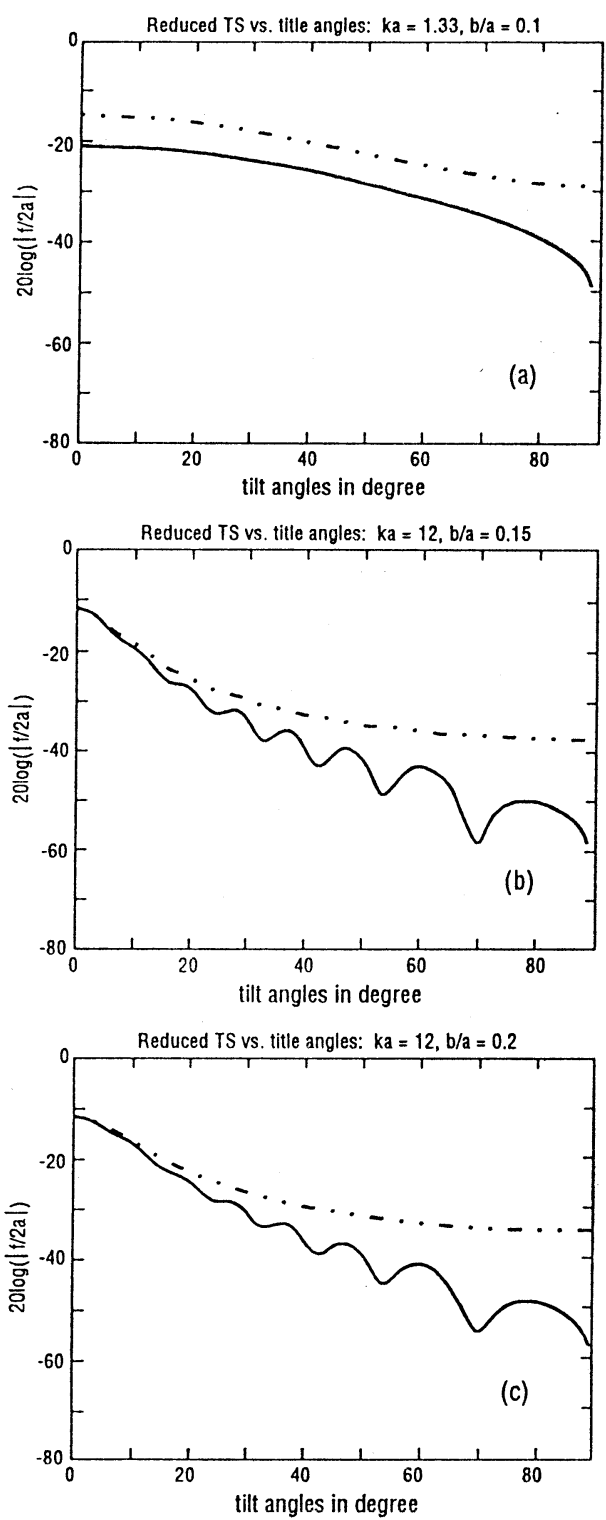

Fig. 4 Reduced TS vs. tilt angles for different aspect ratios and $k a$. In the plots, the solid line is from Eq. (17) and the dotted-dashed lines are from Fig. 4 in Furusawa. ${ }^{1)}$

in Fig. 2 of Clay and Horn, the axis of the swimbladder of cod D is slightly tilted at -9 degree. We consider the normal incidence with respect to the fish body as Clay and Horne did. We plot the reduced scattering amplitude (swimbladder) $|f| L \mid$ 


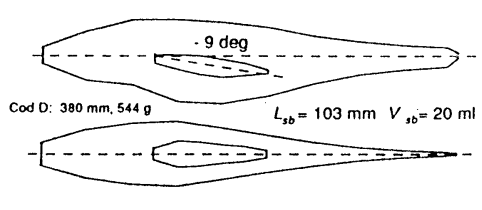

(a)

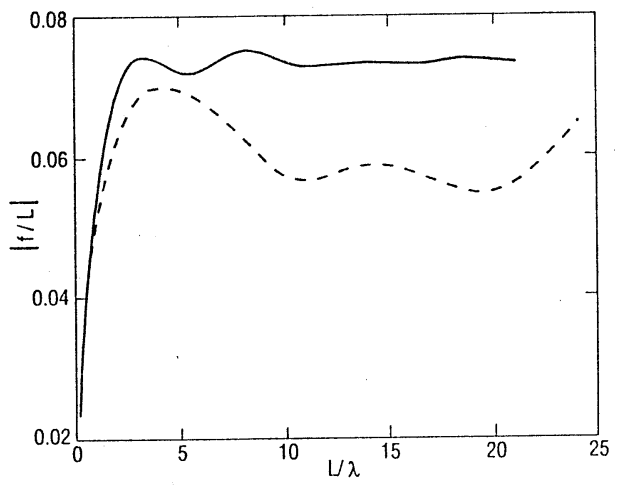

(b)

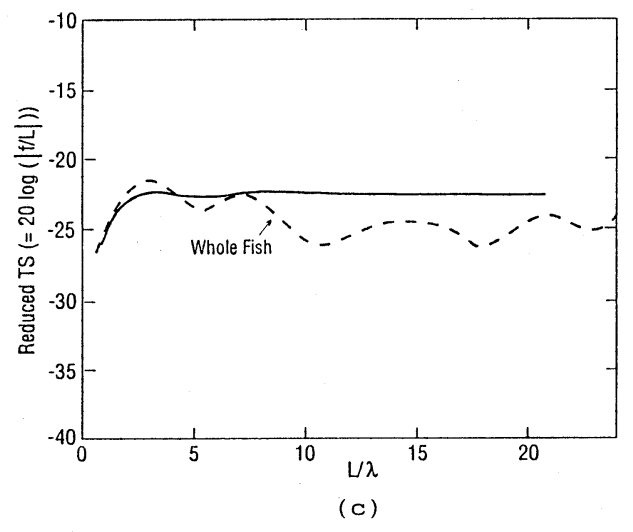

Fig. 5 Comparison with Clay and Horne $^{11)}$ : a) Atlantic cod D from Fig. 2 of Clay and Horne; b) Reduced scattering amplitude for the swimbladder of Atlantic cod D. The solid line is from the present model and the dashed line is from Fig. 6(a) in Ref. 11). c) Reduced TS vs. $L / \lambda$, as compared with Clay and Horne's result. The ray-Kirchhoff result from Fig. 6(d) in Ref. 11) is shown by the dashed line.

vs. $L / \lambda$ in Fig. 5(b). The figure shows that the present model predicts higher scattering amplitude than that of Clay and Horne. The maximum difference is about $30 \%$, occurring at $L / \lambda=18$. At low $L / \lambda$ values, the two models match well. We also plot the reduced TS $v s . L / \lambda$ in Fig. 5(c). The result of Clay and Horne (Fig. 6(d) in Ref. 11)) for the whole fish is also presented in Fig. 5(c). Here $\lambda$ is the acoustic wavelength. We find that our result reasonably agrees with that of Clay and Horne for $L / \lambda$ as low as 0.75 (or $k a \sim 1$ ) and up to 7. However, discrepancy occurs for larger $L / \lambda$, where the present model is expected to agree with the exact prolate spheroid model according to the results in the last subsection. For larger values of $L / \lambda$, a maximum difference of $3 \mathrm{~dB}$ occurs at a bout $L / \lambda=10$ and all of our calculated values are slightly higher than that of Clay and Horne for $L / \lambda>7$.

Now we consider an indirect comparison with the target strength measurements of Nakken and Olsen. ${ }^{16)}$ The experimental data have been compared with the theoretical values of Clay and Horne in Ref. 11). Experimental data were collected with $38 \mathrm{kHz}$ sonar. The wavelength is about $4 \mathrm{~cm}$. According to the above discussions, the present model yields comparable results to that from Clay and Horne for $L / \lambda<7$. This means that the present model can predict more or less the comparable results to what Clay and Horne have obtained for fish length ranging from $0.75 \times 4$ to $7 \times 4$, i.e. 3 to $28 \mathrm{~cm}$. Eye-inspection of Fig. 9 in Ref. 11) shows that in this length range the model of Clay and Horne does give a good match with the experimental data, implying that the present model may also give a good match in this length range. For $L / \lambda>7$, our model yields higher TS values than Clay and Horne's model. As a matter of fact, the measured values are indeed slightly higher than the theoretical values of Clay and Horne for $L / \lambda>7$ (See Fig. 9 in Ref. 11)). It should be pointed out that both diagrams in Figs. 5(b) and (c) are plotted with $L$ being fixed. This is slightly different from the case of the measurements, where the wavelength $\lambda$ is fixed. So we do not intend to compare directly our results with the experimental data at this moment.

Nevertheless we see that our calculated TS of the swimbladder generally follow the trend of that of the whole fish calculated by Clay and Horne. Thus the present study may also confirm that the swimbladder is the most dominant scattering part in a fish body.

We can also calculate the maximum TS by using a general formula proposed by Furusawa. ${ }^{1)}$ The formula states that the maximum TS can be es- 
timated by

$$
T S_{\max }=10 \log \left(a^{2} / 4\right),
$$

where, as before, $a$ is the major radius of the equivalent spheroid corresponding to a fish swimbladder. As surveyed in Furusawa, ${ }^{1)} a$ can be assumed to be 0.17 of the fork length of a fish having a closed swimbladder. In such case, the maximum TS can be calculated from

$$
T S_{\mathrm{max}}=-61.4+20 \log L,
$$

where $L$ is fish fork length in $\mathrm{cm}$. Then the maximum reduced TS can be evaluated as $-21.4 \mathrm{~dB}$, which seems to be reasonable as compared to the maximum value of $-22.4 \mathrm{~dB}$ in Fig. 5(c). The small difference can be accounted for by the fact that Eq. (21) is derived for normal incidence. In the present case, the swimbladder of Atlantic cod $D$ is tilted slightly at -9 degree. Therefore the maximum reduced TS is expected to be smaller than $-21.4 \mathrm{~dB}$ that is for the normal incidence.

\section{CONCLUSIONS}

In conclusion, in this paper we presented a simplified model to calculate the target strength of a fish swimbladder at high frequencies. The theory is stemmed from those developed by Junger ${ }^{9)}$ and Stanton $^{10)}$ for an elongated scatterer. The comparisons were made with two other models. It was shown that the model agrees well with the exact prolate spheroid model for high frequencies and with the ray-Kirchhoff model in an intermediate frequency range. Brief comparisons with experimental data were also made. One merit of the present model is that it generally requires fewer parameter measurements than many other models. Another merit of this model is that it requires little computation time. Therefore it can be easily incorporated into other software which utilizes the information of fish target strength. Since most fish do have elongated swimbladder and most echo sounders are operated at relatively high frequencies, the present model can facilitate target strength calculations and therefore will see many applications. However, it should be noted that the present model may not be appropriate for incidence at large tilted angles. The moral is that while the accurate modeling of fish target strength, incorporating detailed physiological aspects of fish, remains to be a difficult task in fisheries acoustics, it may be more practical to be able to estimate the general trend of target strength by using much simplified models. The present model study can partially accomplish this goal.

\section{ACKNOWLEDGEMENTS}

We thank Drs. Y. Miyanohana and K. Sawada for many useful and stimulating discussions. Ms. P. M. Kimber at IOS is thanked for preparing the figures. The work was completed when $\mathrm{Ye}$ was invited by Drs. M. Furusawa and M. Miyanohana for an one-month visit to the National Research Institute of Fisheries Engineering, Fisheries Agency of Japan. Ye sincerely thanks Drs. Furusawa, Miyanohana and Sawada for their heart felt hospitality and friendships. Ye also thanks fellow Canadian researchers Drs. G. A. McFarlane, R. Kieser and D. M. Farmer for their efforts in making his trip to Japan possible. The work was supported by a fund for the Canada-Japan fisheries acoustics project, awarded by the Science and Technology Agency of Japan. The support from the Department of Fisheries and Oceans of Canada is also greatly acknowledged by $\mathrm{Ye}$.

\section{REFERENCES}

1) M. Furusawa, "Prolate spheroidal models for predicting general trends of fish target strength," J. Acoust. Soc. Jpn. (E) 9, 13-24 (1988).

2) M. Furusawa, Y. Miyanohana, M. Ariji, and $Y$. Sawada, "Prediction of Krill target strength by liquid prolate spheroid model," Fish. Sci. 60, 261265 (1994).

3) I. B. Andreeva, "Scattering of sound by air bladders of fish in deep sound-scattering ocean layers," Sov. Phys. Acoust. 10, 17-20 (1964).

4) B. S. McCartney and A. R. Stubbs, "Measurement of the target strength of fish in the dorsal aspect, including swimbladder resonance," in Proc. Int. Symp. on Biological Sound Scattering in the Ocean, G. B. Farquhar, Ed. (Govt. Printing Office, Washington, D.C., 1970), 180-211.

5) R. H. Love, "Resonant acoustic scattering by swimbladder-bearing fish," J. Acoust. Soc. Am. 64, 571-580 (1978).

6) Z. Ye and D. M. Farmer, "Acoustic scattering from swimbladder fish at low frequencies," J. Acoust. Soc. Am. 96, 951-956 (1994).

7) K. Foote, "Importance of the swimbladder in acoustic scattering by fish: A comparison of gadoid and mackerel target strengths," J. Acoust. Soc. Am. 67, 2084-2089 (1980).

8) K. Foote, "Rather high frequency sound scattering by swimbladder fish," J. Acoust. Soc. Am. 78, 688700 (1985). 


\section{Z. YE and M. FURUSAWA: MODELING OF FISH TARGET STRENGTH}

9) M. C. Junger, "Scattering by slender bodies of revolution," J. Acoust. Soc. Am. 72, 1954-1956 (1982).

10) T. K. Stanton, "Sound scattering by cylinders of finite length. I. Fluid cylinders," J. Acoust. Soc. Am. 83, 55-63 (1988).

11) C.S. Clay and J. K. Horne, "Acoustic models of fish: The Atlantic cod (Gadus morhus)," J. Acoust. Soc. Am. 96, 1661-1668 (1994).

12) R. J. Urick, Principles of Underwater Sound (McGraw-Hill, New York, 1983).

13) J. R. Mentzer, Scattering and Diffraction of Radio Waves (Pergamon, New York, 1955).

14) S. McClatchie, J. Alsop, Z. Ye, and R. Combs, "Consequence of swimbladder model choice and fish orientation to target strength of three New Zealand fish species," submitted to ICES J. Mar. Sci.

15) M. A. Do and A. M. Surti, "Estimation of dorsal aspect target strength of deep water fish using a simple model of swimbladder backscattering," J. Acoust. Soc. Am. 87, 1588-1596 (1990).

16) O. Nakkken and K. Olsen, "Target strength measurements of fish," Rapp. P.-v. Rèun. Cons. Int. Explor. Mer. 170, 52-69 (1977).

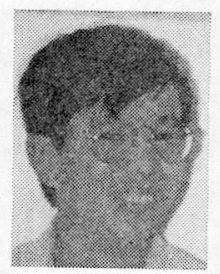

Zhen Ye received B.Sc. degree in Biology from Peking University in 1984. In 1986 he went to study the theoretical physics at the University of Alberta, Canada. He obtained the $\mathrm{Ph} . \mathrm{D}$ in theoretical condensed-matter physics in 1991. In 1991 and 1992 he was a post doctoral fellow with Canadian Centers of Excellence in Molecular and Interfacial Dynamics at the University of Ottawa. Since 1993, he has been with the Institute of Ocean Sciences, $\mathrm{BC}$ Canada. He has been working a wide range of physical problems and recently he also began to work on the problems of ocean acoustics such as fisheries acoustics.

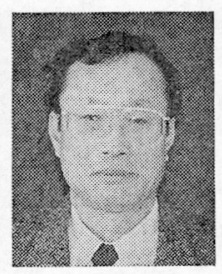

Masahiko Furusawa was born in 1944. He received the B. Eng. degree in electrical engineering and the Dr. Eng. degree from Tokyo Institute of Technology, Tokyo, in 1967 and 1989, respectively. Since 1968 he has been working at National Research Institute of Fisheries Engineering. He is a member of the Acoustical Society of Japan, the Japanese Society of Fisheries Science, the Marine Acoustics Society of Japan, and Institute of Electronics, Information and Communication Engineering of Japan. 